\title{
CHAINS IN PARTIALLY ORDERED SETS
}

\author{
OYSTEIN ORE
}

1. Introduction. Dedekind [1] in his remarkable paper on Dualgruppen was the first to analyze the axiomatic basis for the theorem of Jordan-Hölder in groups. Recently there have appeared a considerable number of investigations in this field; one should mention the contributions by Birkhoff [2], Klein-Barmen [3, 4], Uzkow [5], George [6], Kurosch [7], and the author [8, 9]. Most of these papers deal particularly with the conditions which it is necessary to impose upon a structure or lattice in order to assert that the chains between two elements have the same length.

In the present paper the general problem of comparing chains in partially ordered sets is considered. It seems remarkable and somewhat surprising that one can formulate a theorem about chains in arbitrary partially ordered sets which contains the theorem of JordanHölder as a special case when applied to more restricted systems. This theorem yields for partially ordered sets, hence by specialization also for structures, a necessary and sufficient condition for the chains to have equal lengths.

2. Simple cycles. Let us indicate briefly the terminology which we shall use. The basic partially ordered set to be investigated shall be denoted by $P$. An element $x$ in $P$ lies between two elements $a \supset b$ when $a \supset x \supset b$ and properly between them when the two possibilities $a=x$ and $b=x$ are excluded. An element $a$ is prime over $b$ and $b$ is prime under $a$ when $a \supset b$ and there are no elements properly between $a$ and $b$. A chain is an ordered subset of $P$. A finite chain between $a$ and $b$ has the form

$$
a=a_{0} \supset a_{1} \supset \cdots \supset a_{n-1} \supset a_{n}=b
$$

where $n$ is the length of the chain. A chain is complete when it is not possible to intercalate further terms in it. Through transfinite induction one can prove that any chain is contained in some complete chain.

Now let $C_{1}$ and $C_{2}$ be two complete chains joining two elements $a \supset b$ in such a manner that they have no elements in common except the end points $a$ and $b$. Such two chains shall be said to form a simple cycle when the following condition is fulfilled:

Simple cycle. There shall exist no elements properly between $a$ and $b$

Received by the editors December 5, 1942. 
containing or being contained in two different elements $c_{1}$ and $c_{2}$ belonging to $C_{1}$ and $C_{2}$, respectively.

The two chains $C_{1}$ and $C_{2}$ shall be called the components of the simple cycle they constitute.

To clarify the definition of a simple cycle let us observe that it is tacitly assumed that $c_{1}$ and $c_{2}$ are different from the end points $a$ and $b$ of the cycle. The definition clearly excludes that any $c_{1}$ in $C_{1}$ contains a $c_{2}$ in $C_{2}$ or conversely.

We shall denote by $C_{b}^{a}$ some complete chain from $a$ to $b$. If $C_{b}^{a}$ passes through the two elements $m \supset n$ we shall write $C_{n}^{m}$ for the part of the chain connecting $m$ with $n$. Now let $D_{n}^{m}$ be some other complete chain between $m$ and $n$ so that a new chain $D_{b}^{a}$ may be formed from $C_{b}^{a}$ by replacing $C_{n}^{m}$ by $D_{n}^{m}$. When in this case the two chains $C_{n}^{m}$ and $D_{n}^{m}$ together form the components of a simple cycle we shall say that $D_{b}^{a}$ has been obtained from $C_{b}^{a}$ by a simple deformation. Finally we shall say that two arbitrary chains $C_{b}^{a}$ and $D_{b}^{a}$ between $a$ and $b$ are related and one has been obtained by deformation from the other when one can proceed from one to the other by a series of successive simple deformations.

3. Related chains. By means of these concepts we can prove the following theorem which is basic for the comparison of chains in partially ordered sets :

Theorem 1. Let $P$ be a partially ordered set in which the chains between two elements have a bounded length. Then any two complete chains between two elements $a \supset b$ are related.

Proof. The theorem is evidently true when $a$ is prime over $b$. In this case the longest chain between $a$ and $b$ has the length 1 . The theorem may therefore be proved by means of induction with respect to the length of the longest complete chain connecting the two elements $a \supset b$. We assume that the theorem holds for all pairs of elements $a^{\prime} \supset b^{\prime}$ for which the maximal chain has a length less than $n$ and prove it for any pair $a \supset b$ for which a maximal chain $C_{b}^{a}$ has the length $n$. We shall have to show that if $D_{b}^{a}$ is any other complete chain connecting $a$ and $b$ then $D_{b}^{a}$ can be obtained from $C_{b}^{a}$ by a series of simple deformations.

One can immediately exclude the possibility of $C_{b}^{a}$ and $D_{b}^{a}$ having elements in common other than $a$ and $b$ because in this case the desired result follows from the induction assumption. In the case where $C_{b}^{a}$ and $D_{b}^{a}$ form a simple cycle the result is trivially true. It may therefore be assumed for instance that there exists an element $m$ properly 
between $a$ and $b$ such that $m$ contains an element $c$ in $C_{b}^{a}$ and an element $d$ in $D_{b}^{a}$. Correspondingly there exist complete chains

$$
E_{m}^{a}, \quad F_{c}^{m}, G_{d}^{m}
$$

Since the chain

$$
E_{m}^{a}+F_{c}^{m}
$$

joins $a$ to $c$ and since the maximal length between $a$ and $c$ is less than $n$ it follows by the induction assumption that $C_{c}^{a}$ and the chain (2) are related. Thus also $C_{b}^{a}$ and the chain

$$
C^{\prime}=E_{m}^{a}+F_{c}^{m}+C_{b}^{c}
$$

are related. But the two chains

$$
F_{c}^{m}+C_{b}^{c}, \quad G_{d}^{m}+D_{b}^{d}
$$

are also related as a consequence of the induction so that $C^{\prime}$ and the chain

$$
C^{\prime \prime}=E_{m}^{a}+G_{d}^{m}+D_{b}^{d}
$$

between $a$ and $b$ are related. Finally one sees that $C^{\prime \prime}$ must be related to

$$
D_{b}^{a}=D_{d}^{a}+D_{b}^{d}
$$

so that the theorem is proved.

As a referee of this paper Professor S. MacLane observed that Theorem 1 holds also when one assumes only that the chains between any two elements are finite. A proof of this fact will be published subsequently. This gives a more direct proof of Theorem 2 .

4. The main chain theorem. To obtain the analogue of the JordanHölder theorem that any two complete chains between $a$ and $b$ have the same length it is obviously necessary that the two components in a simple cycle have the same length. But conversely, if this is the case the length of a chain does not change by simple deformations, hence related chains have the same length. From Theorem 1 one concludes therefore that if the chains in the partially ordered set are bounded in length, the necessary and sufficient condition for the Jordan-Hölder theorem to hold is that the two components in a simple cycle have the same length. We shall not formulate this as a theorem since we shall derive the stronger result:

THEOREM 2. Let $P$ be a partially ordered set in which a chain between 
two elements is always finite. Then the necessary and sufficient condition that any two complete chains connecting two elements $a \supset b$ have the same length is that the two components in a simple cycle have the same length. In this case the two complete chains are always related.

Proof. The condition is obviously necessary. To prove the sufficiency we observe first that the theorem is true when $a$ is prime over $b$. Again we can use induction, but this time we shall prefer to use induction with respect to the length of the shortest complete chain connecting the two elements $a \supset b$. This procedure corresponds more closely to the ordinary proof of the theorem of Jordan-Hölder in groups and structures. We assume that the theorem holds for all pairs of elements $a^{\prime} \supset b^{\prime}$ which can be joined by a complete chain of length less than $n$ and prove it for any pair $a \supset b$ which can be joined by a complete chain $C_{b}^{a}$ of length $n$. We shall show that if $D_{b}^{a}$ is any other complete chain connecting $a$ and $b$ then $D_{b}^{a}$ can be obtained from $C_{b}^{a}$ by a series of simple deformations.

As before we can exclude the case when $C_{b}^{a}$ and $D_{b}^{a}$ have elements in common other than $a$ and $b$ and also the case where $a$ and $b$ form a simple cycle, since then the result is true by assumption. It may be assumed therefore as previously that there exists an element $m$ properly between $a$ and $b$ such that $m$ contains an element $c$ in $C_{b}^{a}$ and an element $d$ in $D_{b}^{a}$. Correspondingly there exist complete chains (1). Since the chain (2) joins $a$ to $c$ and since the chain $C_{c}^{a}$ has shorter length than $n$ it follows by the induction assumption that $C_{c}^{a}$ and the chain (2) are related. Thus also $C_{b}^{a}$ and the chain (3) are related and have the length $n$. But then the two elements $m \supset b$ are connected by a complete chain of shorter length than $n$ so that the two chains in (4) are related and have the same length according to the induction assumption. Consequently the two chains in (3) and (5) must be related and have the same length $n$. From this result one concludes finally that the two chains

$$
E_{m}^{a}+G_{d}^{m}, \quad D_{d}^{a}
$$

must have a length shorter than $n$, so that they are related and we have shown that $D_{b}^{a}$ and (5) are related and have the length $n$. This concludes the proof since we have shown successively that the chains $C_{b}^{a}, C^{\prime}$ in (3), $C^{\prime \prime}$ in (5) and $D_{b}^{a}$ are related and have the same length.

In Theorem 2 the finiteness condition for all chains in $P$ is not absolutely necessary. One can prove the following somewhat more general result: 
TheOREM 3. Let $P$ be a partially ordered set with the property that when at least one of the components in a simple cycle has a finite length then both of them have the same length. If two elements $a \supset b$ in $P$ can be connected by a complete finite chain then all complete chains between a and $b$ are related and have the same length.

The proof is a simple extension of the proof of Theorem 2. One uses induction with respect to the length $n$ of the shortest complete chain connecting $a \supset b$. With the same notations as before let $C_{b}^{a}$ be the finite complete chain of length $n$ between $a$ and $b$, and $D_{b}^{a}$ some complete chain to be compared with $C_{b}^{a}$. As before one can assume that the two chains have no elements in common and that they do not form a simple cycle. Consequently there exists an element $m$ and the corresponding chains (1). By the induction assumption the chain (2) must be finite and related to $C_{c}^{a}$, consequently the chains $C_{b}^{a}$ and $C^{\prime}$ in (3) are related and have the same finite length. By the same argument one passes from $C^{\prime}$ to $C^{\prime \prime}$ in (5) and again from $C^{\prime \prime}$ to $D_{b}^{a}$.

5. The quadrilateral condition. The theorems derived in the preceding express results on the comparison of chains in partially ordered sets which appear to be about as general as one can expect to obtain them. In most applications where such theorems have been derived they have been obtained under considerably stronger conditions. These conditions are in many cases included in the condition:

Quadrilateral condition. When $a$ and $b$ are two different elements in a partially ordered set, both contained in some element $u$ and both prime over the element $d$, then there shall exist at least one element $m$ also contained in $u$ and prime over both $a$ and $b$.

The geometric interpretation of this condition on the basis of the diagram of the partially ordered set is simply that when the two sides $(a, d)$ and $(b, d)$ occur in that part of the diagram which is below $u$, there shall also exist two other sides $(m, a)$ and $(m, b)$ in the same part of the diagram completing the quadrilateral $(a, d, b, m)$. Theorem 2 gives immediately this result:

THEOREM 4. Let $P$ be a partially ordered set satisfying the quadrilateral condition. The chains between any two elements $a \supset b$ are supposed to be finite. Then any two complete chains between two elements $a \supset b$ have the same length and one can be obtained from the other by successively replacing two consecutive sides $\left(a_{1}, a_{2}\right)$ and $\left(a_{2}, a_{3}\right)$ by two other sides $\left(a_{1}, b_{2}\right)$ and $\left(b_{2}, a_{3}\right)$ in a quadrilateral.

Proof. Under the given conditions every simple cycle must be a quadrilateral. 
It is possible to extend Theorem 4 to partially ordered sets in which the finiteness condition does not hold. It then takes the following form:

ThEOREM 5. Let $P$ be a partially ordered set satisfying the quadrilateral condition. If in $P$ there exist two complete finite chains connecting two elements $a \supset b$ then both chains are related and have the same length.

Proof. Except for one additional difficulty one can use the arguments of the ordinary proof of the Jordan-Hölder theorem. Let

$$
\begin{aligned}
& a \supset a_{1} \supset \cdots \supset a_{n-1} \supset a_{n}=b, \\
& a \supset b_{1} \supset \cdots \supset b_{r-1} \supset b_{r}=b
\end{aligned}
$$

be the two complete chains connecting $a$ and $b$. As before we use induction and assume that the theorem is true for all pairs connected by complete chains of length less than $n$. As a consequence one can assume that $a_{n-1} \neq b_{r-1}$. From the quadrilateral condition follows the existence of an element $m$ contained in $a$ and prime over $a_{n-1}$ and $b_{r-1}$. Corresponding to (1) we introduce the complete chains

$$
E_{m}^{a}, \quad F_{a_{n-1}}^{m}, \quad G_{b,-1}^{m}
$$

where the last two have the length 1 . If one can now assume that the chain $E_{m}^{a}$ may be taken to be of finite length, the arguments used in the proof of Theorem 1 can be applied to show that both chains in (7) are related and have the same length.

It remains therefore only to establish the existence of a finite complete chain between $a$ and $m=m_{n-2}$. If by chance $m_{n-2}=a_{n-2}$ this is trivial. But when $m_{n-2} \neq a_{n-2}$ there exists by the quadrilateral condition some element $m_{n-3}$ contained in $a$ and prime over $a_{n-2}$ and $m_{n-2}$. Again the finite chain exists when $m_{n-3}=a_{n-3}$. If this is not the case there is an element $m_{n-4}$ prime over $m_{n-3}$ and $a_{n-3}$. Clearly the repetition of this process must eventually give a finite chain between $a$ and $m$.

It should be noted that in Theorem 5 there is no statement about the infinite chains between $a$ and $b$ and the existence of infinite chains is by no means excluded.

6. Weak quadrilateral condition. The quadrilateral condition may be weakened in the following manner:

Weak quadrilateral condition. When $a$ and $b$ are distinct elements prime over $d$ there shall exist at least one element $m$ prime over $a$ and $b$. 
This condition is no longer sufficient to prove that any two finite complete chains between two elements $a \supset b$ in the partially ordered set have the same length. It is however sufficient to prove that certain special chains have the same length. This is expressed in the theorem:

THEOREM 6. Let $P$ be a partially ordered set with a universal element $u$ containing all others and satisfying the weak quadrilateral condition. If there exist two finite complete chains between $u$ and some element a then both chains have the same length and are related.

The proof may be omitted since it involves only a slight modification of the proof of Theorem 5. For the case where $P$ is a partially ordered set in which all chains from $u$ to arbitrary elements $a$ are finite, the fact that all chains have the same length is a result which has recently been established by Newman [10].

7. Application to structures. The preceding theory may now be specialized to the case of structures or lattices. It is clear that in any structure $\Sigma$ both the quadrilateral condition and the weak quadrilateral condition take the form of the so-called

Birkhoff condition. When $a$ and $b$ are prime over their cross-cut $a \cap b$ the union $a \cup b$ shall be prime over $a$ and $b$.

When this condition is satisfied in a structure, Theorem 5 gives the main result that finite complete chains between two elements $a \supset b$ are related and have the same length. This is even a little more than the usual formulation for structures satisfying the Birkhoff condition since one ordinarily assumes that all chains between two elements are finite.

But the main Theorem 1 and its consequences in Theorems 2 and 3 go so much further that it is possible to use them to formulate a necessary and sufficient condition for the equality of the lengths of chains in a structure. Let us consider first the criterion for a simple cycle in a structure. We denote by $C_{b}^{a}$ and $D_{b}^{a}$ two complete chains connecting $a$ and $b$. Then one sees that these two chains are the components of a simple cycle if and only if for every pair of elements $c$ and $d$ different from $a$ and $b$ and belonging to $C_{b}^{a}$ and $D_{b}^{a}$, respectively, one has

$$
c \cup d=a, \quad c \cap d=b .
$$

This form for the definition of a simple cycle makes it natural to introduce the concept of

Cyclic structure. A structure is cyclic when it consists of two chains 
$C$ and $D$ connecting a universal element $a$ with a zero element $b$ such that

$$
c \cup d=a, \quad c \cap d=b
$$

for all elements $c$ and $d$ in $C$ and $D$, respectively.

In other words a cyclic structure is a structure whose elements form a single simple cycle. Let us also say that a cyclic substructure $S$ of a structure $\Sigma$ is complete in $\Sigma$ when the two chains in $\Sigma$ defining $S$ are complete. Thus the simple cycles in a structure are the complete cyclic substructures.

We can now state the main theorem on the comparison of the lengths of chains in structures as a direct consequence of Theorem 2:

THEOREM 7. Let $\Sigma$ be a structure in which the chains between two elements are finite. The necessary and sufficient condition that all complete chains in $\Sigma$ between two elements $a$ and $b$ have the same length is that in any complete cyclic substructure the two defining chains always have the same length.

8. Application to groups. To conclude let us mention one further problem which is connected with this theory of chains. The theorem of Jordan-Hölder for principal chains (each subgroup in the chain normal in the whole group) in groups follows from the theory of Dedekind structures. The ordinary Jordan-Hölder theorem for composition series (each subgroup normal in the preceding) is however not so directly a consequence of the structure theory. This has been observed by various authors and extensions of the abstract theory have been proposed to remedy this deficiency (Uzkow, George, Kurosch). Let us remark that the preceding theory is sufficiently general to solve this difficulty. The partially ordered set of all subgroups occurring in a composition series of the group satisfies the (dual) quadrilateral condition and its complete chains are the composition series in the group, hence they are all related and of the same length. [In detail: If $A \supset B$ are two composition groups, that is, both occur in some composition series, there exists a composition series containing both. When $G \supset B_{1} \supset \cdots \supset B_{n} \supset B \supset \cdots$ is the composition series containing $B$ one obtains the composition series from $A$ to $B$ explicitly as

$$
A \supseteq B_{1} \cap A \supseteq \cdots \supseteq B_{n} \cap A \supseteq B .
$$

The quadrilateral condition expresses only that if $M_{1}$ and $M_{2}$ are maximal normal subgroups of $A$ then the intersection $M_{1} \cap M_{2}$ is a maximal normal subgroup both of $M_{1}$ and $M_{2}$.] 


\section{BIBLIOGRAPHY}

1. R. Dedekind, Math. Ann. vol. 53 (1900) pp. 371-403; Collected works, vol. 2, pp. 236-271.

2. G. Birkhoff, On the combination of subalgebras, Proc. Cambridge Philos. Soc. vol. 29 (1933) pp. 441-464.

3. F. Klein-Barmen, Ueber ausgeglichene Verbände, Math. Zeit. vol. 42 (1937) pp. 411-418.

4. - - Birkhoffsche und harmonische Verbände, ibid. vol. 42 (1937) pp. 58-81.

5. A. I. Uzkow, On the Jordan-Hölder theorem, Rec. Math. (Mat. Sbornik) N.S. vol. 4 (1938) pp. 31-42.

6. E. George, Ueber den Satz von Jordan-Hölder-Schreier, J. Reine Angew. Math. vol. 180 (1939) pp. 110-120.

7. A. G. Kurosch, The theorem of Jordan-Hölder in arbitrary structures, Memorial volume to D. A. Grave, Moscow, 1940, pp. 110-116.

8. O. Ore, On the foundation of abstract algebra. I, Ann. of Math. (2) vol. 36 (1935) pp. $406-437$.

9. - On the theorem of Jordan-Hölder, Trans. Amer. Math. Soc. vol. 41 (1937) pp. 266-275.

10. M. H. A. Newman, On theories with a combinatorial definition of "equivalence," Ann. of Math. (2) vol. 43 (1942) pp. 223-242.

YALE UNIVERSITY 